\title{
Benefit of hospital admission for detecting serious adverse events among emergency department patients with syncope: a propensity-score-matched analysis of a multicentre prospective cohort
}

\author{
Rohin J. Krishnan MSc, Muhammad Mukarram MBBS MPH, Bahareh Ghaedi MSc, Marco L.A. Sivilotti MD MSc, \\ Natalie Le Sage MD PhD, Justin W. Yan MD MSc, Paul Huang MD, Mona Hegdekar MD, Eric Mercier MD MSc, \\ Marie-Joe Nemnom MSc, Lisa A. Calder MD MSc, Andrew D. McRae MD PhD, Brian H. Rowe MD MSc, \\ George A. Wells MSc PhD, Venkatesh Thiruganasambandamoorthy MBBS
}

Cite as: CMAJ 2020 October 13;192:E1198-1205. doi: 10.1503/cmaj.191637

\begin{abstract}
BACKGROUND: The benefit of hospital admission after emergency department evaluation for syncope is unclear. We sought to determine the association between hospital admission and detection of serious adverse events, and whether this varied according to the Canadian Syncope Risk Score (CSRS).
\end{abstract}

METHODS: We conducted a secondary analysis of a multicentre prospective cohort of patients assessed in the emergency department for syncope. We compared patients admitted to hospital and discharged patients, using propensity scores to match $1: 1$ for risk of a serious adverse event. The primary outcome was detection of a serious adverse event in hospital for admitted patients or within 30 days after emergency department disposition for discharged patients.

RESULTS: We included 8183 patients, of whom 743 (9.1\%) were admitted; $658 / 743$ (88.6\%) were matched. Admitted patients had higher odds of detection of a serious adverse event (odds ratio [OR] 5.0, 95\% confidence interval [CI] 3.3-7.4), nonfatal arrhythmia (OR $5.1,95 \% \mathrm{Cl} 2.9-8.8)$ and nonarrhythmic serious adverse event (OR 6.3, 95\% Cl 2.9-13.5). There were no significant dif- ferences between the 2 groups in death (OR 1.0, 95\% Cl 0.4-2.7) or detection of ventricular arrhythmia (OR $2.0,95 \% \mathrm{Cl}$ 0.7-6.0). Differences between admitted and discharged patients in detection of serious adverse events were greater for those with a CSRS indicating medium to high risk $(p=0.04)$.

INTERPRETATION: Patients with syncope were more likely to have serious adverse events identified within 30 days if they were admitted to hospital rather than discharged from the emergency department. However, the benefit of hospital admission is low for patients at low risk of a serious adverse event. yncope accounts for $1 \%-2 \%$ of all emergency department visits. ${ }^{1}$ Although syncopal events are usually benign, a serious underlying condition (e.g., arrythmia, myocardial infarction, severe hemorrhage) is identified within 30 days in about $10 \%$ of patients. ${ }^{2}$ Such underlying conditions are potentially life-threatening, and some, such as ventricular arrhythmia, can be fatal within minutes. The morbidity and mortality associated with these underlying conditions represent serious adverse events. No interventions can be offered to patients with syncope until a serious adverse event manifests as part of the clinical course. For 3\%-5\% of patients, such an event is not identified until after the index emergency department disposition. ${ }^{3,4}$ It is a major challenge for emergency department physicians to identify those at risk for serious adverse events and decide an appropriate disposition plan (hospital admission or discharge). ${ }^{5}$ 
Previous studies examining the role of hospital admission for patients with syncope have given contradicting results, ${ }^{6-8}$ leading to a lack of guidance from professional societies regarding the benefit of admission for syncope. ${ }^{1,9}$ As a result, the decision about emergency department disposition for patients with syncope remains unstructured, with wide variations even among similar institutions across Canada. ${ }^{10}$

Although it seems obvious that more serious adverse events will be identified in hospital owing to increased surveillance that occurs during hospital admission, surprisingly, a recent US study showed no benefit of admission for detection of serious adverse events, even among older ( $\geq 60 \mathrm{yr}$ ) emergency department patients with syncope. ${ }^{11}$ Hence, it remains unclear whether there is a benefit to hospital admission and, if yes, the specific types of serious adverse events identified in hospital. ${ }^{12}$ A randomized controlled trial could provide an answer; however, ethical considerations make such a trial currently unfeasible. ${ }^{13}$

We recently completed 2 large prospective studies in which patients with syncope were admitted to hospital or discharged from the emergency department based on physician judgment, allowing comparison of detection of serious adverse events between the 2 groups. ${ }^{3}$ Therefore, the primary objective of this study was to determine whether a greater incidence of serious adverse events was detected among patients admitted to hospital while in hospital than in a comparable group of patients who were discharged. As a secondary objective, we explored whether the association between emergency department disposition and occurrence of a serious adverse event varied according to risk for a serious adverse event, as determined with the Canadian Syncope Risk Score (CSRS). ${ }^{3}$ We hypothesized that the clinical benefit in the form of increased detection of serious adverse events with hospital admission compared to discharge would be higher with increasing CSRS.

\section{Methods}

We conducted a secondary analysis of data from 2 prospective cohort studies carried out at 11 sites (Appendix 1, available at www.cmaj.ca/lookup/doi/10.1503/cmaj.191637/tab-related -content) across Canada from September 2010 to April 2018. ${ }^{3}$ Adult patients ( $\geq 16 \mathrm{yr}$ ) who presented to the emergency department within 24 hours of experiencing syncope were included. We excluded patients who were unable to provide their history because of a language barrier, cognitive impairment, or drug or alcohol intoxication, and those who presented with loss of consciousness for more than 5 minutes, change in mental status from baseline, witnessed seizure, major trauma necessitating admission or head trauma leading to loss of consciousness. Patients with a serious underlying condition identified during the index emergency department evaluation were also excluded from this analysis. We collected patient characteristics, medical history, emergency department vital signs, syncope details, results of investigations, final emergency department diagnostic impression and disposition. ${ }^{4,14}$

\section{Outcomes}

The primary outcome was a serious adverse event (Appendix 2, available at www.cmaj.ca/lookup/doi/10.1503/cmaj. 191637 /tab-related-content) that was identified in hospital after the decision to admit was made for patients who were admitted, or within 30 days among those discharged directly from the emergency department. We defined a serious adverse event as the occurrence or identification of any serious underlying condition or its sequelae, including death (due to known or unknown cause), ventricular arrhythmia, nonfatal arrhythmic serious adverse events (including nonventricular arrhythmia or procedural intervention to treat arrhythmia) and nonarrhythmic serious adverse events (e.g., myocardial infarction, structural heart disease, aortic dissection, pulmonary embolism, pulmonary hypertension, subarachnoid hemorrhage, severe hemorrhage or any other serious condition causing the syncope).

\section{Statistical analysis}

We used descriptive statistics with means and standard deviations (SDs) or medians and interquartile ranges for continuous variables as appropriate. We reported frequencies and proportions to describe categoric variables and used the $\chi^{2}$ test to compare proportions. We report the distribution of serious adverse events among patients who were admitted (both during admission and after discharge) and those who were discharged from the emergency department.

We used propensity score matching to examine whether the probability of detecting serious adverse events was higher for admitted patients than for those who were discharged directly from the emergency department. All patients included in this study are identified by the term "original cohort," and the subgroup matched by means of propensity score as the "matched cohort." Variables used to generate the propensity score included demographic characteristics (age, sex), clinical correlates (blood pressure, prodrome symptoms, vasovagal predisposition, syncope diagnosis, abnormal electrocardiogram, elevated troponin level, and history of heart disease, hypertension or diabetes) and setting (arrival to the emergency department by ambulance and hospital site) (Appendix 3, available at www.cmaj.ca/lookup/doi/10.1503/ cmaj.191637/tab-related-content). We chose the model for generating the propensity score when the distribution of baseline characteristics between admitted and discharged patients with the same propensity score was similar. ${ }^{14,15}$

We matched admitted patients to the nearest control patient (1:1 greedy matching without replacement) based on the logit of the propensity score. Matches were restricted to a caliper width equal to $20 \%$ of the SD of the logit of the propensity score. ${ }^{16}$ We deemed matching quality to be sufficient if the standardized differences of baseline variables between admitted and discharged patients were less than $10 \%$. We then used logistic regression to assess the association of emergency department disposition and detection of serious adverse events within the matched cohort, and we report adjusted odds ratios (ORs) with 95\% confidence intervals (Cls). We calculated 
robust standard errors, taking into account clustering between matched pairs.

We also performed the following additional analyses. We assessed the fragility of the primary outcome to hidden bias by varying the OR for differential treatment assignment and computing a corresponding Mantel-Haenszel statistic and $p$ value. ${ }^{17}$ For this analysis, the magnitude of the OR, denoted by $\gamma$, ranged from 1 to 5 in increments of 0.5 . The $\gamma$ value with a corresponding $p$ value $>0.05$ represents the strength of an unmeasured confounder needed to render the primary results nonsignificant.

To compare our results to those of a similar previous study, ${ }^{11}$ we restricted the sample to adults aged 60 or older. Admitted and discharged patients in this subgroup were subsequently rematched without replacement, and a new matched cohort was created. We explored the association of emergency department disposition and detection of serious adverse events in the original cohort using propensity score weighting with inverse probability treatment weights. ${ }^{18}$ In addition, we estimated the number of additional serious adverse events that could have potentially occurred among discharged patients lost to 30-day follow-up using predicted probabilities from the CSRS ${ }^{3}$ (Appendix 4, available at www.cmaj.ca/lookup/doi/10.1503/cmaj.191637 /tab-related-content).

For the secondary objective (benefit of hospital admission based on CSRS), we conducted a multivariable logistic regression with the original cohort to determine the interaction between CSRS score and admission for detection of a serious adverse event, adjusting for variables that are not CSRS predictors (age, sex, hypertension, diabetes, diastolic blood pressure, arrival by ambulance, prodrome symptoms and hospital site; area under the curve 0.91; Hosmer-Lemeshow goodness-of-fit $p$ value $=0.8$ ) (Appendices 3 and 4$)$. We calculated the difference in predicted probabilities for detection of a serious adverse event between admitted and discharged patients within each CSRS level. We used Stata statistical software (StataCorp) for analysis.

\section{Ethics approval}

As both parent prospective studies were observational, with no patient interventions, the ethics committees at the study sites approved the study with the requirement of only verbal consent.

\section{Results}

Of 8183 patients in the original cohort (Figure 1, Table 1), $743(9.1 \%)$ were admitted for syncope and $293(3.6 \%, 95 \% \mathrm{Cl}$ $3.2 \%-4.0 \%$ ) experienced a 30 -day serious adverse event after the index emergency department disposition (Table 2). Of those admitted, 196 (26.4\%) experienced a 30-day serious adverse event, $169(22.7 \%)$ during the index hospital stay and $27(3.6 \%)$ after. The remaining serious adverse events were identified in 97 patients $(1.3 \%)$ who were discharged from the emergency department $(p<0.001$ for serious adverse events identified in hospital in the admitted group $v$. outside hospital in the discharged group).

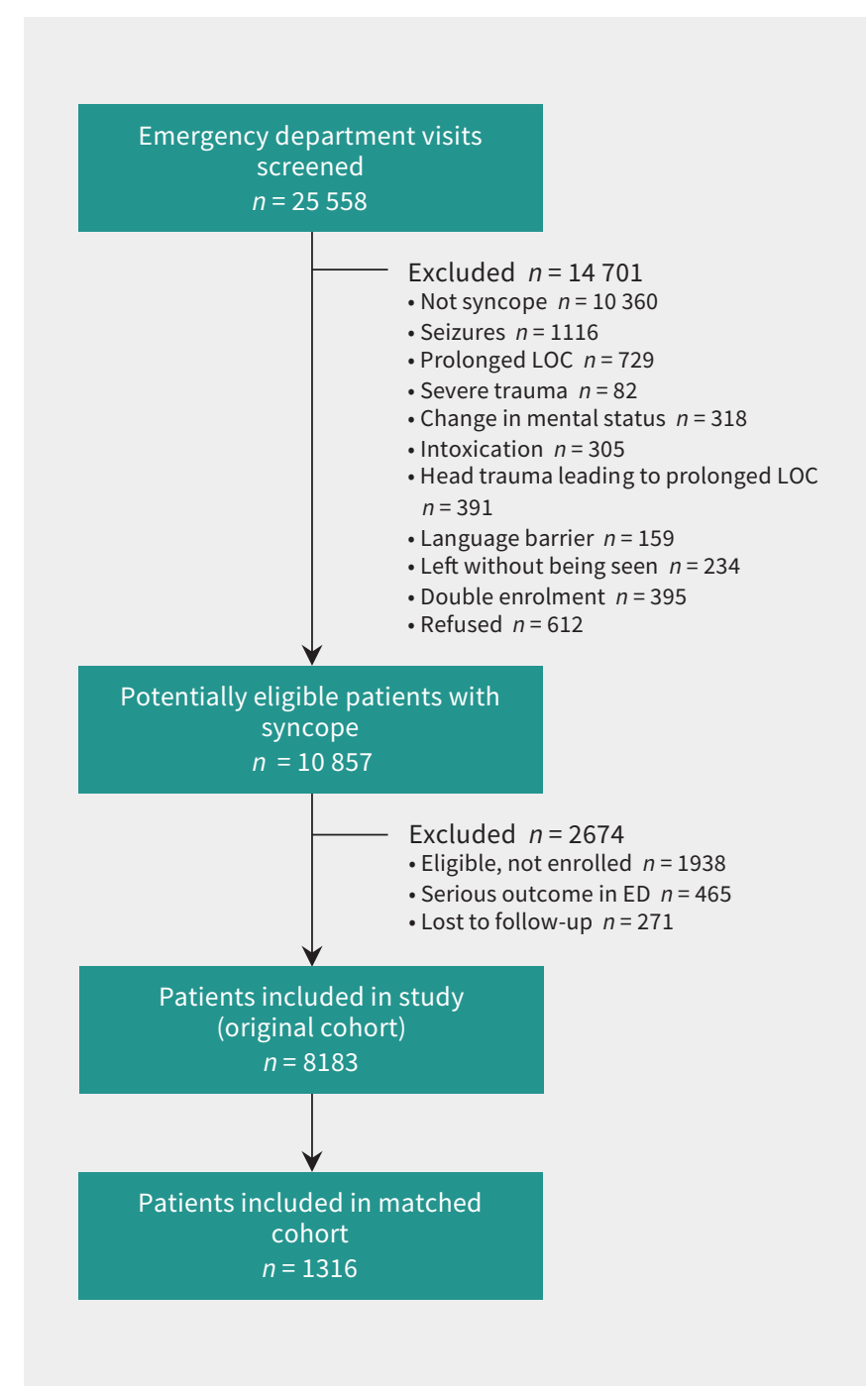

Figure 1: Flow chart showing patient selection. Note: $E D=$ emergency department, $L O C=$ loss of consciousness.

In the original cohort, compared to discharged patients, admitted patients were older, predominantly male, more likely to be transported to the emergency department by ambulance, more likely to have abnormal blood pressure values, less likely to have prodromal symptoms and vasovagal predisposition, more likely to be diagnosed with cardiac syncope, more likely to have elevated troponin levels and abnormal electrocardiogram features, and more likely to belong to the medium, high or very high CSRS risk categories (Table 1 ).

Of the 743 patients (9.0\%) admitted for syncope, 658 $(88.6 \%)$ could be matched to a discharged patient. The unmatched patients were older and predominantly male, and a higher proportion had higher-risk characteristics (Appendix 5, Supplemental Table S1, available at www.cmaj.ca/lookup/ doi/10.1503/cmaj.191637/tab-related-content). After matching, the characteristics, including risk of a serious adverse event, were similar between the admitted and discharged groups (Table 1). The standardized difference was less than 10\% for the baseline variables in the matched cohort (Table 1). 
Table 1: Characteristics of patients in the original cohort and in that matched by propensity score

\begin{tabular}{|c|c|c|c|c|c|c|}
\hline \multirow[b]{2}{*}{ Characteristic } & \multicolumn{3}{|c|}{$\begin{array}{l}\text { Original cohort, no. (\%) of patients* } \\
\qquad n=8183\end{array}$} & \multicolumn{3}{|c|}{$\begin{array}{l}\text { Matched cohort, no. (\%) of patients* } \\
\qquad n=1316\end{array}$} \\
\hline & $\begin{array}{l}\text { Admitted to } \\
\text { hospital } \\
n=743\end{array}$ & $\begin{array}{l}\text { Discharged } \\
n=7440\end{array}$ & $\begin{array}{l}\text { Standardized } \\
\text { difference, \% }\end{array}$ & $\begin{array}{l}\text { Admitted to } \\
\text { hospital } \\
n=658\end{array}$ & $\begin{array}{l}\text { Discharged } \\
n=658\end{array}$ & $\begin{array}{l}\text { Standardized } \\
\text { difference, \% }\end{array}$ \\
\hline Age, mean $\pm S D$, yr & $70.6 \pm 17.5$ & $51.8 \pm 22.7$ & 92.8 & $69.4 \pm 17.9$ & $70.6 \pm 17.2$ & 5.9 \\
\hline Female sex & $299(40.2)$ & 4209 (56.6) & 33.1 & $278(42.2)$ & $291(44.2)$ & 4.0 \\
\hline Arrival by ambulance & $553(74.4)$ & $4661(62.6)$ & 25.6 & 480 (72.9) & $490(74.5)$ & 3.3 \\
\hline \multicolumn{7}{|l|}{ Blood pressure } \\
\hline $\begin{array}{l}\text { Mean systolic blood pressure } \\
\text { during ED stay < } 90 \mathrm{~mm} \mathrm{Hg} \text { or } \\
>180 \mathrm{~mm} \mathrm{Hg}\end{array}$ & $215(28.9)$ & $745(10.0)$ & 49.2 & $179(27.2)$ & $186(28.3)$ & 2.8 \\
\hline $\begin{array}{l}\text { Mean diastolic blood pressure } \\
\text { during ED stay }<50 \mathrm{~mm} \mathrm{Hg} \text { or } \\
>110 \mathrm{~mm} \mathrm{Hg}\end{array}$ & $188(25.3)$ & $678(9.1)$ & 44.0 & $160(24.3)$ & $155(23.6)$ & 2.1 \\
\hline $\begin{array}{l}\text { Prodrome (e.g., dizziness, light- } \\
\text { headedness, vision changes, nausea } \\
\text { or vomiting) }\end{array}$ & $366(49.3)$ & $5701(76.6)$ & 59.6 & $342(52.0)$ & $347(52.7)$ & 1.6 \\
\hline $\begin{array}{l}\text { Vasovagal predisposition (e.g., warm } \\
\text { crowded place, prolonged standing, } \\
\text { fear, emotion or pain)† }\end{array}$ & $120(16.2)$ & $3544(47.6)$ & 71.8 & $118(17.9)$ & $103(15.6)$ & 5.2 \\
\hline \multicolumn{7}{|l|}{ Final ED syncope diagnosis } \\
\hline Vasovagal syncope & $108(14.5)$ & $4274(57.4)$ & 100.0 & $107(16.3)$ & $107(16.3)$ & 0.0 \\
\hline Cardiac syncope & $216(29.1)$ & $236(3.2)$ & 75.2 & $146(22.2)$ & $131(19.9)$ & 6.6 \\
\hline Age-related comorbidities & $464(62.4)$ & $2265(30.4)$ & 67.7 & & & \\
\hline History of hypertension & $424(57.1)$ & $2053(27.6)$ & 62.5 & $361(54.9)$ & $360(54.7)$ & 0.3 \\
\hline History of diabetes & $176(23.7)$ & $674(9.1)$ & 40.3 & $146(22.2)$ & $155(23.6)$ & 3.8 \\
\hline History of heart disease $\ddagger \S$ & 356 (47.9) & $1249(16.8)$ & 70.5 & $296(45.0)$ & $298(45.3)$ & 0.7 \\
\hline $\begin{array}{l}\text { Elevated troponin level }(>99 \text { th } \\
\text { percentile of population) } \dagger\end{array}$ & 155 (20.9) & $248(3.3)$ & 55.8 & $100(15.2)$ & $98(14.9)$ & 1.0 \\
\hline \multicolumn{7}{|l|}{ Electrocardiogram characteristics } \\
\hline QRS duration $\geq 130$ ms $\dagger$ & $130(17.5)$ & $334(4.5)$ & 42.5 & $100(15.2)$ & $100(15.2)$ & 0 \\
\hline QRS axis $<30^{\circ}$ or $>110^{\circ} \dagger$ & $147(19.8)$ & $580(7.8)$ & 35.3 & $115(17.5)$ & $107(16.3)$ & 3.6 \\
\hline Corrected QT interval > $480 \mathrm{~ms}^{\dagger}$ & $156(21.0)$ & $399(5.4)$ & 47.5 & $125(19.0)$ & $126(19.1)$ & 0.5 \\
\hline \multicolumn{7}{|l|}{ Syncope risk category } \\
\hline Very low & $51(6.9)$ & $3518(47.3)$ & 102.2 & $51(7.8)$ & $54(8.2)$ & 1.1 \\
\hline Low & $134(18.0)$ & $2483(33.4)$ & 35.7 & $132(20.1)$ & $154(23.4)$ & 7.8 \\
\hline Medium & $324(43.6)$ & $1155(15.5)$ & 64.6 & $317(48.2)$ & $298(45.3)$ & 6.7 \\
\hline High & $149(20.0)$ & $213(2.9)$ & 56.0 & $119(18.1)$ & $104(15.8)$ & 7.5 \\
\hline Very high & $85(11.4)$ & $63(0.8)$ & 45.2 & $39(5.9)$ & $48(7.3)$ & 5.9 \\
\hline Serious adverse event & $169(22.8)$ & $97(1.3)$ & - & $133(20.2)$ & $32(4.9)$ & - \\
\hline \multicolumn{7}{|c|}{ 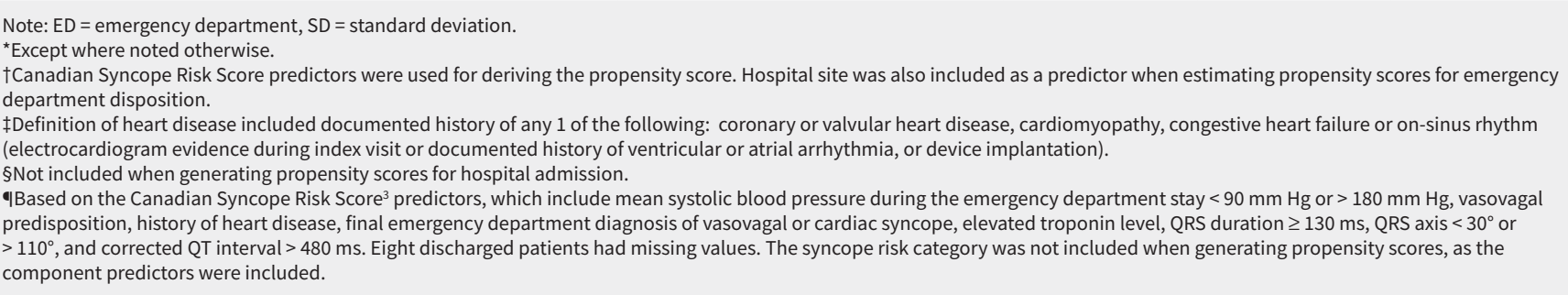 } \\
\hline
\end{tabular}




\section{Table 2: Thirty-day serious adverse events among 8183 emergency department patients} admitted to hospital or discharged

\begin{tabular}{|c|c|c|c|c|}
\hline \multirow[b]{2}{*}{ Outcome* } & \multicolumn{3}{|c|}{$\begin{array}{l}\text { Admitted; no. (\%) of patients } \\
\qquad n=743\end{array}$} & \multirow{2}{*}{$\begin{array}{c}\text { Discharged; } \\
\text { no. (\%) of patients } \\
n=7440\end{array}$} \\
\hline & Total & $\begin{array}{l}\text { During index } \\
\text { hospital stay }\end{array}$ & $\begin{array}{l}\text { After index } \\
\text { hospital stay }\end{array}$ & \\
\hline Serious adverse event & $196(26.4)$ & $169(22.7)$ & $27(3.6)$ & $97(1.3)$ \\
\hline Death & $19(2.6)$ & $10(1.3)$ & $9(1.2)$ & $15(0.2)$ \\
\hline Ventricular arrhythmia & $20(2.7)$ & $18(2.4)$ & $2(0.3)$ & $9(0.1)$ \\
\hline $\begin{array}{l}\text { Death or ventricular } \\
\text { arrhythmia }\end{array}$ & $36(4.8)$ & $26(3.5)$ & $10(1.3)$ & $23(0.3)$ \\
\hline Nonfatal arrhythmia & $106(14.3)$ & $92(12.4)$ & $14(1.9)$ & $51(0.7)$ \\
\hline $\begin{array}{l}\text { Nonarrhythmic serious } \\
\text { adverse event }\end{array}$ & $62(8.3)$ & $57(7.7)$ & $5(0.7)$ & $30(0.4)$ \\
\hline
\end{tabular}

Table 3: Odds ratios for serious adverse events during hospital admission in admitted patients and within 30 days in discharged patients*

$$
\text { No. }(\%) \text { of patients }
$$

\begin{tabular}{|c|c|c|c|}
\hline Outcome & $\begin{array}{l}\text { Admitted to } \\
\text { hospital } \\
n=658\end{array}$ & $\begin{array}{c}\text { Discharged } \\
n=658\end{array}$ & $\begin{array}{c}\text { OR† } \\
(95 \% \mathrm{CI}) \ddagger\end{array}$ \\
\hline Serious adverse event & $133(20.2)$ & $32(4.9)$ & $5.0(3.3-7.4)$ \\
\hline $\begin{array}{l}\text { Death (due to unknown } \\
\text { and known cause) }\end{array}$ & $8(1.2)$ & $8(1.2)$ & $1.0(0.4-2.7)$ \\
\hline Ventricular arrhythmia & $10(1.5)$ & $5(0.8)$ & $2.0(0.7-6.0)$ \\
\hline $\begin{array}{l}\text { Death or ventricular } \\
\text { arrhythmia }\end{array}$ & $17(2.6)$ & $12(1.8)$ & $1.4(0.7-3.0)$ \\
\hline Nonfatal arrhythmia & $74(11.2)$ & $15(2.3)$ & $5.1(2.9-8.8)$ \\
\hline $\begin{array}{l}\text { Nonarrhythmic serious } \\
\text { condition§ }\end{array}$ & $47(7.1)$ & $8(1.2)$ & $6.3(2.9-13.5)$ \\
\hline
\end{tabular}

Note: $\mathrm{Cl}=$ confidence interval, $\mathrm{OR}=$ odds ratio.

*Among the 1316 matched emergency department patients with syncope.

tCalculated by logistic regression analysis within the matched cohort.

‡Clustering among matched pairs was taken into account when calculating standard errors.

$\S$ Described in Appendix 2.

The odds of detecting a serious adverse event in hospital for admitted patients compared to discharged patients over the 30 days following emergency department disposition are reported in Table 3. The OR for detecting any serious adverse event in those admitted to hospital was 5.0 (95\% Cl 3.3-7.4). Differences between the 2 groups in death (OR 1.0, 95\% Cl 0.42.7) or identification of ventricular arrhythmia (OR $2.0,95 \% \mathrm{Cl}$ 0.7-6.0) were not statistically significant. However, the odds of detecting nonfatal arrhythmia (OR 5.1, 95\% Cl 2.9-8.8) and nonarrhythmic serious adverse event (OR 6.3, 95\% Cl 2.9-13.5) were significantly higher for admitted patients during their hospital stay than for discharged patients over 30 days. The distribution of nonfatal arrythmia between the 2 groups in the matched cohort are presented in Appendix 5, Supplemental Table S2.

Analysis for violations of the conditional independence assumption was robust; an unobserved predictor would need to influence the odds of admission or discharge assignment by a factor of 3.5 to render the primary outcome nonsignificant (Appendix 5, Supplemental Table S3). We also replicated the primary findings with inverse probability treatment weighting in the original cohort (Appendix 5, Supplemental Table S4).

Among older patients ( $\geq 60 \mathrm{yr}$ ), detection of serious adverse events was significantly higher for admitted patients during their hospital stay than for discharged patients over 30 days (OR 7.7, 95\% Cl 4.8-12.3).

A total of 278 patients were lost to 30 -day follow-up, 7 in the admitted group and 271 in the discharged group. The 7 admitted patients did not have serious adverse events identified while in the hospital. Of the 271 discharged patients, 152 were at very low risk, 90 were at low risk, 25 were at medium risk, 3 were at high risk, and 1 was at very high risk. The increased detection of serious adverse events among admitted patients remained statistically significant $(p<0.001$ for serious adverse events identified in hospital in the admitted group v. outside the hospital in the discharged group), even after we accounted for additional serious adverse events that may have occurred among discharged patients who were lost to follow-up (Appendix 5, Supplemental Table S5).

For the secondary objective (benefit of admission according to the CSRS), after adjustment for variables that were not CSRS predictors, the interaction between emergency department disposition and CSRS was significant in the original cohort $(p=$ 0.04). Overall, in the entire cohort, the difference in detection of serious adverse events among admitted patients while in hospital compared to discharged patients over 30 days was small for 


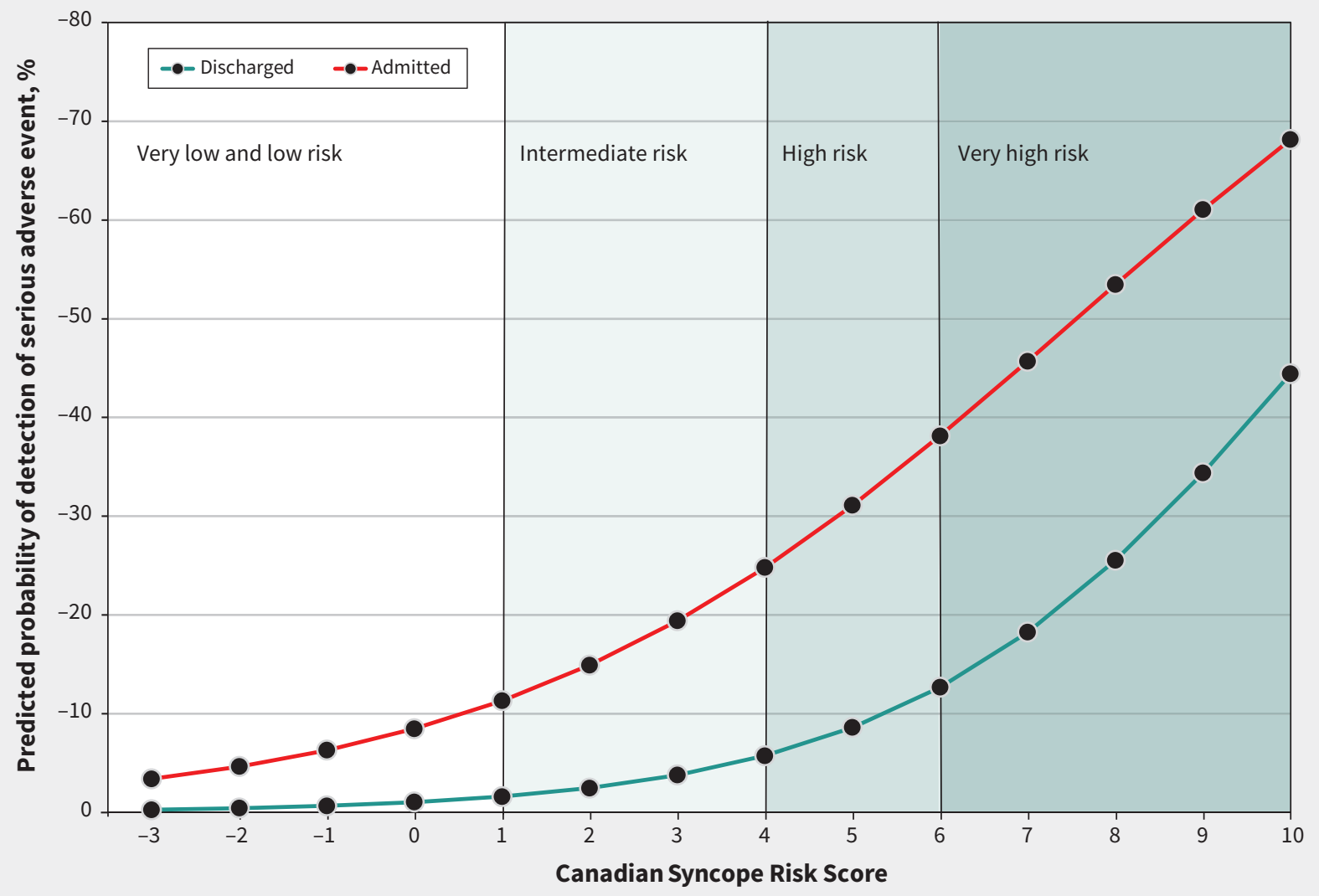

Figure 2: Predicted probability of 30-day serious adverse events based on the Canadian Syncope Risk Score (CSRS) among the 8096 emergency department patients admitted to hospital or discharged. The interaction term between emergency department disposition and CSRS was significant in the multivariable logistic model $(p=0.04)$. Eighty-seven patients were excluded from the multivariable logistic regression analysis owing to missing predictor information.

patients at very low and low risk but more pronounced for those at medium, high or very high risk (Figure 2; Appendix 5, Supplemental Table S6).

\section{Interpretation}

We found that an underlying serious condition was identified in a higher proportion of patients admitted to hospital while they were in hospital than in patients discharged from the emergency department within 30 days of index syncope. The increased detection of serious adverse events was driven by nonfatal arrhythmic and nonarrhythmic serious adverse events, with no differences in rates of death or ventricular arrhythmia between the groups in the matched cohort. Overall, the increased detection of serious adverse events with admission was greater for patients at medium risk or higher for a serious adverse event according to the CSRS.

Our findings contrast with a recent propensity-scorematched analysis of patients in the United States. Probst and colleagues ${ }^{11}$ found no difference in detection of serious adverse events among older ( $\geq 60 \mathrm{yr}$ ) adults with syncope between those admitted and those discharged. One noteworthy finding is the sharp contrast in the proportion of patients admitted to hospital between our Canadian study (9\%) and that of Probst and colleagues (75\%). This highlights an important concern: low thresholds for hospital admission lead to increased admission of patients at low risk. This is likely the reason why Probst and colleagues ${ }^{11}$ observed no difference in detection of serious adverse events between admitted and discharged patients. Taken together, the findings underscore the urgent need for better risk stratification to standardize disposition decisions for syncope. Increased detection of serious underlying conditions with hospital admission will have the most clinical benefit for patients who are at increasingly higher risk to have a serious adverse event identified within 30 days.

Effective emergency department evaluation for syncope requires a pragmatic approach using validated tools, along with shared decision-making with patients and families. For 
example, in our study, we observed increased detection of nonlethal arrythmia with hospital admission. However, identification of nonfatal arrhythmia can also be achieved by lengthy cardiac rhythm monitoring in the outpatient setting. ${ }^{19}$ Such a strategy will increase the proportion of underlying arrhythmias identified at a lower cost compared to hospital admission. In addition, given that the short-term incidence of death and life-threatening ventricular arrhythmia is very low in patients with syncope and is not decreased with hospital admission, patients at low risk according to the CSRS can be discharged. ${ }^{20}$ Patients at medium risk with adequate home support can be discharged and advised to seek care if serious conditions evolve. Patients among whom an evolving condition is suspected or at high or very high risk can be admitted to hospital.

\section{Limitations}

Unobserved variables may have influenced emergency department disposition in our matched cohort. Seventy-five patients (10\%) admitted to hospital could not be matched. Although we found that a significantly higher proportion of admitted patients than discharged patients had nonlethal arrhythmic serious adverse events, this finding is subject to ascertainment bias among those in hospital compared to those discharged.

For the secondary objective, 8 of the 16 variables used for propensity score matching were CSRS predictors. ${ }^{3}$ This posed a challenge, as it was not possible to derive meaningful estimates for the interaction of emergency department disposition and CSRS in the matched cohort. To overcome this limitation, we conducted a multivariable logistic regression in the original cohort, adjusting for predictors not included in the CSRS.

Roughly $18 \%$ ( $n=1938$ ) of patients were eligible but were not included in the cohort. It is likely that attending emergency department physicians were too busy to complete data collection or discharged the patients before data collection. Four percent $(n=328)$ of patients had missing information for electrocardiogram predictors, and 60\% $(n=4950)$ did not have troponin assays completed. As in our previous studies, ${ }^{3}$ those values were assumed to be normal, as the patients with missing variables were young, with fewer comorbidities and more often diagnosed with vasovagal syncope. About $4 \%(n=271)$ of discharged patients were lost to follow-up, and therefore their 30-day outcomes could not be ascertained. Our sensitivity analysis showed that the results were not affected by the additional serious adverse events estimated among discharged patients lost to follow-up.

\section{Conclusion}

We found that an underlying serious condition was identified in a higher proportion of admitted patients during their hospital stay than among discharged patients within 30 days of the emergency department disposition. However, our analysis showed no difference in detection of death or ventricular arrhythmia. These findings underscore the need for robust risk stratification to better optimize health resource use and reduce mismatches between disposition and risk of a serious adverse event. The CSRS may be a useful tool to support making this decision. Patients at low risk according to the CSRS are least likely to have a serious adverse event identified while in hospital.

\section{References}

1. Shen WK, Sheldon RS, Benditt DG, et al. 2017 ACC/AHA/HRS guideline for the evaluation and management of patients with syncope: executive summary - a report of the American College of Cardiology/American Heart Association Task Force on clinical practice guidelines and the Heart Rhythm Society. J Am Coll Cardiol 2017;70:620-63.

2. Reed MJ. Approach to syncope in the emergency department. Emerg Med $J$ 2019;36:108-16.

3. Thiruganasambandamoorthy V, Kwong K, Wells GA, et al. Development of the Canadian Syncope Risk Score to predict serious adverse events after emergency department assessment of syncope. CMAJ 2016;188:E289-98.

4. Quinn J, McDermott D, Stiell I, et al. Prospective validation of the San Francisco Syncope Rule to predict patients with serious outcomes. Ann Emerg Med 2006;47:448-54.

5. Gallagher EJ. Shooting an elephant. Ann Emerg Med 2004;43:233-7.

6. Morag RM, Murdock LF, Khan ZA, et al. Do patients with a negative emergency department evaluation for syncope require hospital admission? J Emerg Med 2004;27:339-43.

7. Shiyovich A, Munchak I, Zelingher J, et al. Admission for syncope: evaluation, cost and prognosis according to etiology. Isr Med Assoc J 2008;10:104-8.

8. Schillinger M, Domanovits H, Mullner M, et al. Admission for syncope: evaluation, cost and prognosis. Wien Klin Wochenschr 2000;112:835-41.

9. Brignole M, Moya A, de Lange FJ, et al.; ESC Scientific Document Group. 2018 ESC guidelines for the diagnosis and management of syncope. Eur Heart $J$ 2018;39:1883-948

10. Thiruganasambandamoorthy V, Taljaard M, Stiell IG, et al. Emergency department management of syncope: need for standardization and improved risk stratification. Intern Emerg Med 2015;10:619-27.

11. Probst MA, Su E, Weiss RE, et al. Clinical benefit of hospitalization for older adults with unexplained syncope: a propensity-matched analysis. Ann Emerg Med 2019;74:260-9.

12. Canzoniero JV, Afshar E, Hedian $\mathrm{H}$, et al. Unnecessary hospitalization and related harm for patients with low-risk syncope. JAMA Intern Med 2015;175: 1065-7.

13. Costantino G, Dipaola $\mathrm{F}$, Solbiati $\mathrm{M}$, et al. Is hospital admission valuable in managing syncope? Results from the STePS study. Cardiol J 2014;21:606-10.

14. Garrido MM, Kelley AS, Paris J, et al. Methods for constructing and assessing propensity scores. Health Serv Res 2014;49:1701-20.

15. Becker SO, Ichino A. Estimation of average treatment effects based on propensity scores. Stata J 2002;2:358-77.

16. Austin PC. Optimal caliper widths for propensity-score matching when estimating differences in means and differences in proportions in observational studies. Pharm Stat 2011;10:150-61.

17. Becker SO, Caliendo M. Sensitivity analysis for average treatment effects. Stata J 2007;7:71-83.

18. Hernán MA, Robins JM. Causal inference: what if. Boca Raton (FL): Chapman \& Hall/CRC; 2020. Available: www.hsph.harvard.edu/miguel-hernan/causal -inference-book/ (accessed 2019 Dec. 1).

19. Thiruganasambandamoorthy V, Rowe BH, Sivilotti MLA, et al. Duration of electrocardiographic monitoring of emergency department patients with syncope. Circulation 2019;139:1396-406.

20. Costantino G, Sun BC, Barbic F, et al. Syncope clinical management in the emergency department: a consensus from the first international workshop on syncope risk stratification in the emergency department. Eur Heart J 2016;37: 1493-8. 
Competing interests: Lisa Calder is a paid employee of the Canadian Medical Protective Association. Venkatesh Thiruganasambandamoorthy received an honorarium and travel expenses for attending a 2-day focus group on syncope sponsored by Medtronic. No other competing interests were declared.

This article has been peer reviewed.

Affiliations: John A. Burns School of Medicine (Krishnan), University of Hawai'i at Mānoa, Honolulu, Hawaii; Ottawa Hospital Research Institute (Krishnan, Mukarram, Ghaedi, Nemnom, Calder, Thiruganasambandamoorthy), The Ottawa Hospital, Ottawa, Ont.; Departments of Emergency Medicine (Sivilotti) and Biomedical and Molecular Sciences (Sivilotti), Queen's University, Kingston, Ont.; Department of Family Medicine and Emergency Medicine (Le Sage, Mercier), Laval University-Centre hospitalier universitaire de Québec - Université Laval Research Centre, Québec, Que.; Division of Emergency Medicine (Yan), Western University, London, Ont.; Department of Emergency Medicine (Huang), University of British Columbia, Vancouver, BC; Department of Emergency Medicine (Hegdekar), University of Manitoba, Winnipeg, Man.; Department of Emergency Medicine (Calder, Thiruganasambandamoorthy) and School of Epidemiology and Public Health (Wells, Thiruganasambandamoorthy), University of Ottawa, Ottawa, Ont.; Departments of Emergency Medicine (McRae) and Community Health Sciences (McRae), University of Calgary, Calgary, Alta.; Department of Emergency Medicine (Rowe) and School of Public Health (Rowe), University of Alberta, Edmonton, Alta.

Contributors: Muhammad Mukarram, Bahareh Ghaedi, Marco Sivilotti, Natalie Le Sage, Justin Yan, Paul Huang, Mona Hegdekar, Eric Mercier, Lisa Calder, Andrew McRae, Brian Rowe and Venkatesh Thiruganasambandamoorthy supervised the conduct of the study including recruitment of patients, data collection and data management. Rohin Krishnan, Muhammad Mukarram, Bahareh Ghaedi, Marco Sivilotti, Natalie Le Sage, Justin Yan, Paul Huang, Mona Hegdekar, Eric Mercier, Lisa Calder, Andrew McRae, Brian Rowe, George Wells and Venkatesh Thiruganasambandamoorthy conceived the study, contributed to the study design and developed the study protocol.Marie-Joe Nemnom and George Wells provided statistical advice on the study design. Rohin Krishnan analyzed the data under the supervision of George Wells. Rohin Krishnan, Marie-Joe Nemnom, George Wells and Venkatesh Thiruganasambandamoorthy interpreted the data. Rohin Krishnan and Venkatesh Thiruganasambandamoorthy drafted the manuscript. All of the authors revised the manuscript critically for important intellectual content, approved the final version to be published and agreed to be accountable for all aspects of the work.

Funding: The first prospective study was funded by the Physicians' Services Incorporated Foundation (09q4017) and the Canadian Institutes of Health Research (CIHR) (MOP-114927). The second prospective study was funded by the Heart and Stroke Foundation of Canada (G-15-0009006) and the Cardiac Arrhythmia Network of Canada (SRG15-P10-001) as part of the Networks of Centres of Excellence. Venkatesh Thiruganasambandamoorthy holds a National New Investigator Award salary award through the Heart and Stroke Foundation of Canada. Brian Rowe's research is supported by CIHR through a Tier I Canada Research Chair in Evidence-based Emergency Medicine from the Government of Canada.

Data sharing: If approved by the study hospitals through data-sharing agreements, unidentified data can be made available for use by other researchers.

Acknowledgements: The authors are deeply indebted to all the patients who participated in 0 this study. They gratefully acknowledge the emergency physicians at the study sites who recruited the patients and the emergency medicine residents who helped in this process. They acknowledge the following members of their research team: Ottawa site: Pam Ladouceur, Sarah Gaudet, Karen Sauve, Dr. Aparna Vaidyanathan, Soo-Min Kim, My-Linh Tran, Sheryl Domingo, Aline Christelle Ishimwe, Faheem Malam, Zein Ahmed, Angela Marcantonio, Connor Monk and Dr. Hina Chaudry; Kingston site: Jane Reid, Laura Goodfellow, Nicole O'Callaghan and Dr. Vlad Latiu; London site: Dr. Melanie Columbus, Kristine Van Aarsen and Dimah Azzam; Québec site: Marilyne Dufresne, Catherine Bédard and Suzy Lavoie; Vancouver site: Rupinder Brar, Vi Ho and Dr. Corinne Hohl; Winnipeg site: Dr. Anne Finlayson, Christine Kennedy and Monica Manhas; and Edmonton site: Pamela Pang and Natalie Runham.

Disclaimers: The funders take no responsibility for the design, conduct, results or interpretations presented here. George Wells is a biostatistical consultant for CMAJ and was not involved in the editorial decision-making process for this article.

Accepted: June 16, 2020

Correspondence to: Venkatesh Thiruganasambandamoorthy, vthirug@ohri.ca 